\title{
1. Demographic change and the lifecourse: an introduction
}

\author{
Jane Falkingham, Maria Evandrou and Athina Vlachantoni
}

\section{$1.1 \quad$ WHY FOCUS ON DEMOGRAPHIC CHANGE AND THE LIFECOURSE?}

Over the past two decades the lifecourse perspective has become an indispensable tool in the study of demographic change, driven by - and reflected in - advances in both theory and data. Theoretical perspectives on the lifecourse continue to highlight the importance in our understanding of demographic change of such principles as the timing and sequencing of events, the role of human agency and the significance of linked lives; while the establishment and increasing sophistication of longitudinal datasets, both within and across country contexts, has made the application of the lifecourse perspective possible in empirical research. Demographers across the globe have responded enthusiastically to the methodological challenges associated with studying demographic events over time, with appreciating the role of the individuals' environment in shaping their choices and decisions, and with operationalising key concepts such as the duration and timing of demographic events. The result has been a step-change in our understanding of the causes and consequences of population change, and a vast expansion in the evidence base informing contemporary policy debates on a global scale; be it on family formation and dissolution, migration, inequalities of different forms, and the complex quest for improving wellbeing at the individual, household and societal level. This volume is a small selection of contributions from demographers around the world on the role of the lifecourse perspective in their research.

The volume is divided into six parts. First, we consider recent theoretical and methodological advances in lifecourse research. We then turn to a discussion of data and how the evolution of key sources of panel and cohort data have fuelled innovation in lifecourse research. The next three parts present research shedding light on family dynamics and living arrangements, health, and migration and mobility over the lifecourse. The sixth and final part illustrates the implications of lifecourse research for policy.

\subsection{THEORETICAL AND METHODOLOGICAL ADVANCES IN LIFECOURSE RESEARCH}

Chapter 2 introduces Part I on theoretical and methodological advances in lifecourse research, and presents the lifecourse cube as an innovative tool for analysing individual lifecourses and interdependencies across three dimensions: time, life domains and levels of analysis (or process levels). This chapter discusses how the complexity embodied in the lifecourse cube can be applied to a range of topics within demographic research, including the dynamics of fertility, mortality and migration. 
Chapter 3 critically discusses lifecourse-sensitive concepts and measures of social inequality, and the usefulness of a lifecourse perspective in the study of social stratification. The authors argue that in times of rapid demographic change, the lifecourse perspective is particularly valuable. The chapter discusses relevant examples, for instance, the combination of family instability, on the one hand, and more precarious employment, on the other hand, which could increase the prevalence of socio-economic inequalities for individuals in all age groups.

The incorporation of the lifecourse perspective in empirical research has opened up a range of methodological possibilities in the field of demography, particularly in terms of longitudinal analysis. Chapter 4 provides a succinct discussion of such methodologies, distinguishing between event-based methods (such as event history analysis) and holistic methods (such as sequence analysis). This chapter also discusses the types of research questions which can be addressed with different methodologies, and the strengths and weaknesses of each method, within the broader framework of lifecourse-sensitive longitudinal analysis.

\subsection{DATA AND INNOVATION IN LIFECOURSE RESEARCH}

A key driver of the increasing application of the lifecourse perspective within empirical analysis of demographic change has been the expansion in the availability of appropriate data that allow researchers to capture the complexity of individual lifecourses. In Part II, Chapter 5 introduces the dataset established by the Generation and Gender Programme (GGP), which is one of the fundamental datasets allowing for cross-national comparative and longitudinal analysis in areas such as intergenerational exchange and support. This chapter highlights selected cases of research where the GGP data have been used, for example, the study of family dynamics and childbearing, and transitions to adulthood.

Chapter 6 outlines the characteristics of another key data source for research applying the lifecourse perspective - the Survey of Health, Ageing and Retirement in Europe (SHARE). This chapter discusses in particular the life history element of the survey, presenting examples from six domains where recent analysis has made a contribution to extending our understanding of demographic change and the lifecourse: childhood circumstances and later life wellbeing, health and healthcare, labour market and occupation, income and wealth, work and retirement, and the impact of European history on individuals and societies alike.

Chapter 7 explores the growing body of evidence about family transitions, including the family structure, partnership formation and dissolution and childbearing, drawing on data from the 1958 and the 1970 British birth cohort studies. Through the discussion in this chapter, the authors highlight the importance of the historical context in which the cohorts grew up for understanding differences across cohorts. They discuss the opportunities for future research in this area, for example, the study of the 'living apart together' phenomenon, and the cohorts' transitions into later life experiences such as grandparenthood.

Applying a lifecourse perspective, Chapter 8 discusses the ways in which data from the UK Household Longitudinal Study (or Understanding Society) have been analysed to shed new light on different stages of the lifecourse (childhood, adulthood, older age). In particular, the authors illustrate the importance of context (time and place) and how these have featured in recent empirical analysis. 


\subsection{FAMILY DYNAMICS AND LIVING ARRANGEMENTS OVER THE LIFECOURSE}

The previous chapters have highlighted the importance of data for understanding demographic change. Part III now focuses in more detail on family dynamics and living arrangements over the lifecourse. In Chapter 9 the authors use data from the UK Millennium Cohort Study and the US Fragile Families Study in order to compare and contrast the experiences of married and unmarried parents during the first five years of children's lives. Their comparative analysis shows that cohabiting families with young children tend to be more unstable, vulnerable and impoverished than families of married couples; and single mothers and their children tend to have lower levels of wellbeing than married or cohabiting families, although the latter result is not consistent across the two contexts and different indicators.

Chapter 10 examines intermarriage in Australia using marriage registration and census data, focusing on the interethnic pattern of marriages in Australia and the marriage behaviour of migrants to Australia. This chapter argues that restrictive migration policies have a direct and negative impact on union formation patterns among migrants to Australia, and that there is a clear gender dimension in such phenomena, with women being more likely to be secondary applicants or sponsored by an Australian-born partner. Within a lifecourse perspective, the chapter illustrates how key demographic events such as marriage and partnership formation can be facilitated or disrupted by migration.

Focusing on population change and housing across the lifecourse, Chapter 11 discusses the macro-level relationship between population change and the housing system, considering the interdependencies between age composition, household trends and housing demand. This chapter outlines how the lifecourse perspective can be applied to the study of what is termed 'housing demography', illustrating the links between residential mobility and family formation.

\subsection{HEALTH OVER THE LIFECOURSE}

One of the greatest achievements of the twentieth century was the extension of life expectancy. Understanding the influences on health at different stages of the lifecourse, how an individual's health at one stage influences his or her health later in life and how to narrow the gap in healthy life expectancy across social groups is critical if we are to consolidate and continue these gains. In Part IV, focusing on the importance of early life, Chapter 12 provides a succinct summary of current research and thinking on the effects of long and short birth spacing on the wellbeing of children, using a range of health, educational and socio-economic outcome variables across different points of the lifecourse. Highlighting the importance of the context in which individual trajectories are studied, the chapter draws on evidence from research embedded in the lifecourse perspective and aimed at understanding such effects at various levels of social and economic development.

Chapter 13 then critically reviews evidence which links work, family and health across the lifecourse, drawing on the three British birth cohort studies (1946, 1958, 1970). This chapter highlights the role of lifecourse-sensitive analysis in understanding how the timing of events affects individuals' wellbeing. The chapter centres around women who combine work and family, and its effect on their health: are women who combine work and family healthier as 
a result of combining employment with a stable partnership and parenthood, or is the association the result of healthier women being more likely to enter the workforce and have children, or a combination of bi-directional processes? The chapter argues that women who spend long periods of time out of the labour market to look after home and family end up being less healthy on a range of markers compared with women who combine paid employment with a stable partnership and parenthood, suggesting that there is a cohort effect with such health differences becoming stronger for younger generations of women who are less likely to spend long periods out of the labour market.

\subsection{MIGRATION AND MOBILITY OVER THE LIFECOURSE}

We live in an increasingly mobile world. In Part V, Chapter 14 makes a case for the central role of the lifecourse theory in our understanding of contemporary population mobilities. It uses residential mobility in the UK in order to illustrate the strengths of the lifecourse perspective; for example, in terms of acknowledging the role of human agency in mobility patterns, timing of events and the principle of linked lives. This chapter also includes suggestions for fertile areas of future research, such as the linkage of individual lives to the broader institutional framework where they are situated, exploring for example mobility experiences in a time of recession and austerity.

Chapter 15 contributes to our understanding of the interrelationship between partnership, residential mobility and housing transitions. Using data from the British Household Panel Survey, the authors apply event history analysis to examine residential mobility by partnership status, further distinguishing between separated individuals according to the time since separation. They then use panel regression methods to examine home ownership by partnership status and gender. The chapter shows that married individuals have the lowest mobility rates, and single men and women the highest. Among separated individuals, as the time since separation increases, the mobility rates decrease, with no gender differences in this respect. The chapter concludes with a discussion of the methodological challenges remaining in this area, and suggestions for future research.

Drawing on data from the Dutch population registers, Chapter 16 examines the consequences of European Union (EU) migration for national welfare systems. The chapter focuses on EU migrants from Poland and Bulgaria to the Netherlands in the years after these countries joined the EU, and specifically the impact of labour market restrictions on their welfare access. The authors find that the migrants' lifecourse characteristics, length of stay and labour market status are as important in determining their eligibility to welfare as the relevant national and European frameworks, thereby highlighting the importance of embedding a lifecourse perspective in future analysis in this area.

\section{$1.7 \quad$ POLICY}

Many of the preceding chapters have highlighted the importance of the institutional environment for understanding differences in lifecourse transitions across countries or sub-groups within countries. The final part of this volume focuses more explicitly on the role of policy in influencing demographic change and the implications of population change for policy across 
the lifecourse. Chapter 17 uses data from the Fragile Families and Child Wellbeing Study in the USA in order to critically discuss the impact of different types of welfare state benefits on families with children. The authors describe the bifurcated nature of welfare state transfers by income and family type: poor and fragile families receive income-tested health insurance and housing benefits, while middle- and upper-income, and mostly married-parent, families receive generous employer-provided health benefits and tax-subsidised home ownership benefits. Critical and policy-relevant questions are raised in this chapter about the optimal characteristics of welfare support in the USA, and its role in terms of the economic wellbeing of all families, and especially fragile families.

Elder care for frail older adults in the USA is provided through a variety of channels, including formal and informal providers. Chapter 18 reviews the current framework on paid leave for elder care in the USA, highlighting gaps where research evidence on the impact of different family leave policies is evident. The authors also suggest recommendations for policy reforms and directions for future research, for example, studies documenting the effects of paid family leave on the caregivers' work, health and care provision.

Using data from the SHARE dataset, the authors of Chapter 19 explore the financial situation of retired migrants from the first wave of post-war migration to the European Economic Area (EEA). They find that migrants living in the EEA who stayed in their host country on retirement are at a significantly greater risk of relative poverty than native retired citizens, however, such risk varies depending on the migrants' country of origin, with those from non-EEA countries faring worse. The chapter raises critical questions about the characteristics of different pension systems, and the ways in which migrants' economic wellbeing is affected.

In Chapter 20 the authors present an analysis of the Understanding Society dataset linking individuals' employment history and later life satisfaction, distinguishing between three cohorts (1940-44, 1945-49, 1950-54). Sequence analysis is used in order to group earlier life employment histories into eight clusters representing different work trajectories. The analysis shows that older people in the part-time returning group report the highest life satisfaction, followed by the full-time employed and those who shifted from being full-time employed to self-employed during their working life. Using a lifecourse approach, the authors then conduct path analysis to unravel this relationship further. This reveals that lifecourse employment history has an indirect effect on life satisfaction, mediated by individuals' health pension security and housing tenure. The analysis highlights the complexity of the lifecourse and how different domains are interlinked.

Taken together, the chapters in this volume illustrate the state of the art in research on demographic change and the lifecourse and in doing so underline the necessity for continuous advances in data, methods and theory. We thank all the contributors and hope you enjoy the read. 